\title{
ORIGINAL RESEARCH \\ Intra-Arterial Thrombolysis of Occluded Middle Cerebral Artery by Use of Collateral Pathways in Patients with Tandem Cervical Carotid Artery/ Middle Cerebral Artery Occlusion
}

O. Ozdemir

M. Bussière

A. Leung

I. Gulka

D. Lee

R. Chan

J.D. Spence

D. Pelz

BACKGROUND AND PURPOSE: Cervical internal carotid artery (ICA) occlusion with middle cerebral artery (MCA) embolic occlusion is associated with a low rate of recanalization and poor outcome after intravenous thrombolysis. Prompt revascularization is required to prevent disabling stroke. We report our experience on acute ischemic stroke patients with tandem ICA or MCA occlusions treated with microcathether navigation and intra-arterial thrombolysis by use of collateral pathways including the posterior or anterior communicating arteries, or both pathways.

\begin{abstract}
MATERIALS AND METHODS: We retrospectively identified 8 patients with proximal ICA occlusion associated with MCA embolic occlusions treated with intra-arterial thrombolysis (IA rtPA). Access to the occluded MCA was obtained via catheter navigation through intact collateral pathways, including posterior communicating (PcomA) or anterior communicating (AcomA) arteries, without passing a microcathether through the acutely occluded ICA. We assessed clinical outcomes using modified Rankin scale (mRS) and National Institutes of Health Stroke Scale (NIHSS).
\end{abstract}

RESULTS: Eight patients with a mean age of $57 \pm 4$ years and median NIHSS of 14 were identified. Mean time from stroke onset to intra-arterial thrombolysis was $292 \pm 44$ minutes. The MCA was revascularized completely in 5 of the 8 patients via collateral intra-arterial rtPA administration. All of the patients had a favorable outcome defined as a mRS of $\leq 2$ or more at 1 and 3 months' follow-up after thrombolytic therapy. One patient had an asymptomatic petechial hemorrhage.

CONCLUSION: In this small number of patients with tandem occlusions of the ICA and MCA, intraarterial thrombolysis and recanalization of the MCA by use of collateral pathways to bypass the occluded ICA is a safe and efficacious therapeutic option.

A cute occlusion of middle cerebral artery (MCA) occurs in up to $50 \%$ of patients with occlusion of the internal carotid artery (ICA). ${ }^{1}$ The prognosis of these patients is poor, with high rates of morbidity and mortality. ${ }^{2}$ Intra-arterial thrombolysis can result in better outcomes in patients with acute occlusions of the MCA. ${ }^{3}$ There is currently no clear consensus on the optimal treatment approach for patients with acute ischemic stroke secondary to tandem ICA and MCA occlusions. Several different strategies have been used, including combined intravenous and intra-arterial thrombolysis by microcatheter navigation through the occluded ICA, ${ }^{4}$ mechanical thrombectomy, ${ }^{5,6}$ or angioplasty with or without stent placement of the occluded ICA. ${ }^{7-10}$ These approaches can be time consuming, and the increased complexity of the treatment is associated with increased potential risk for complications. We report our experience on the treatment of pa-

Received September 29, 2007; accepted after revision April 16, 2008

From the Departments of Clinical Neurological Sciences (0.0., M.B., R.C., J.D.S.) and Diagnostic Radiology and Nuclear Medicine (M.B., A.L., I.G., D.L., D.P.), University of Western Ontario, London Health Sciences, University Campus, London, Ontario, Canada.

M.B. has received fellowship funding from the Heart and Stroke Foundation of Canada, AstraZeneca Canada, and a Resident Research Career Development Award from the Schulich School of Medicine \& Dentistry, University of Western Ontario.

Abstract previously presented at: Annual Meeting of the American Society for Neuroradiology, June 9-14, 2007; Chicago, III.

Please address correspondence to Ozcan Ozdemir, MD, Department of Clinical Neurological Sciences, University of Western Ontario, London Health Sciences, University Campus, 339 Windermere Rd, Room A7-030, London, ON, Canada N6A5A5; e-mail: ozcan_99@yahoo. com

DOI 10.3174/ajnr.A1163 tients with acute ischemic stroke with tandem MCA and ICA occlusions treated with microcatheter navigation and intraarterial thrombolysis with use of collateral pathways, including the posterior (PcomA) or anterior communicating (AcomA) arteries. To the best of our knowledge, there have only been 2 previous case reports described in the literature of use of this approach. ${ }^{11,12}$

\section{Materials and Methods}

All patients who underwent endovascular pharmaceutical and/or mechanical thrombolysis between 2001 and 2006 at the London Health Sciences Centre were retrospectively reviewed. Eight patients with tandem ICA and MCA occlusions were treated with intra-arterial thrombolysis via catheter navigation through intact collateral pathways (either the PcomA or AcomA), without passing a microcatheter through the acutely occluded ICA.

All patients presenting with an acute ischemic stroke were evaluated by a neurologist with expertise in stroke. Nonenhanced CT scans were performed in all patients before treatment with thrombolytics. The extent of the baseline noncontrast CT scan lesion was determined by the Alberta Stroke Program Early CT (ASPECT) Score. ${ }^{13}$ Intravenous thrombolytic was administered at the discretion of the stroke neurologist according to published guidelines. ${ }^{14}$ We treated patients with combined intravenous and intra-arterial thrombolytic therapy if a thrombus was identified in the proximal MCA and/or ICA on MR angiography (MRA) or CT angiogram (CTA). Briefly, intravenous rtPA was given within 3 hours of stroke onset. Angiography was then performed, and if a thrombus was identified, recombinant tissue plasminogen activator (rtPA) was given intra-arterially up to a total dose 


\begin{tabular}{|c|c|c|c|c|c|c|}
\hline Case No & Age yr/sex & $\begin{array}{c}\text { Onset to IA } \\
\text { Treatment (min) }\end{array}$ & Site of Occlusion & Collateral Pathway & $\begin{array}{c}\text { Initial } \\
\text { ASPECT }\end{array}$ & $\begin{array}{l}\text { Cause of } \\
\text { Stroke }\end{array}$ \\
\hline Case 1 & $53 / \mathrm{M}$ & 342 & LICA/LMCA:M1 & AcomA & 7 & Atherothrombotic \\
\hline Case 2 & $42 / M$ & 360 & LICA/LMCA:M1 & PcomA & 6 & Unknown \\
\hline Case 3 & $64 / \mathrm{M}$ & 250 & LICA/LMCA:M1 & PcomA & 8 & Atherothrombotic \\
\hline Case 4 & $46 / M$ & 300 & LICA/LMCA:M1 & AcomA & 7 & Unknown \\
\hline Case 5 & $55 / \mathrm{M}$ & 300 & LICA/LMCA:M1 & AcomA & 9 & Cardioembolic \\
\hline Case 6 & $65 / M$ & 250 & LICA/LMCA;M1 & AcomA/PcomA & 8 & Atherothrombotic \\
\hline Case 7 & $68 / \mathrm{F}$ & 300 & LICA/LMCA:M1 & AcomA & 8 & Atherothrombotic \\
\hline Case 8 & $71 / \mathrm{M}$ & 240 & RICA/RMCA:M1 & PcomA & 8 & Atherothrombotic \\
\hline
\end{tabular}

Note:-ASPECT indicates Alberta Stroke Program Early CT score; RICA, right internal carotid artery; LICA, left internal carotid artery; LMCA, left middle cerebral artery; RMCA, right middle cerebral artery; AcomA, anterior communicating artery; PcomA, posterior communicating artery; IA, intra-arterial.

\begin{tabular}{|c|c|c|c|c|c|c|}
\hline Case No & $\begin{array}{r}\text { Initial } \\
\text { NIHSS }\end{array}$ & $\begin{array}{l}\text { 24-Hour } \\
\text { NIHSS }\end{array}$ & $\begin{array}{l}\text { 1-Month Outcome } \\
\text { NIHSS/mRS }\end{array}$ & $\begin{array}{l}\text { 3-Month Outcome } \\
\text { NIHSS/mRS }\end{array}$ & $\begin{array}{c}\text { Thrombolytic Agent Route/ } \\
\text { Dose of rtPA/mg }\end{array}$ & $\begin{array}{l}\text { Post Thrombolysis } \\
\text { TIMI /MCA }\end{array}$ \\
\hline 1 & 8 & 4 & $1 / 1$ & $1 / 1$ & IA 25 & 2 \\
\hline 2 & 15 & 12 & $5 / 2$ & $4 / 2$ & IA 8 & 2 \\
\hline 3 & 21 & 7 & $3 / 1$ & $3 / 1$ & IV 8.7/IA 15 & 3 \\
\hline 4 & 16 & 8 & $5 / 2$ & $5 / 2$ & IV 7.5/IA 15 & 1 \\
\hline 5 & 14 & 2 & $2 / 1$ & $1 / 0$ & IV 49.2/IA 2 & 2 \\
\hline 6 & 18 & 14 & $5 / 2$ & $3 / 1$ & IV 60/IA 15 & 2 \\
\hline 7 & 8 & 1 & $1 / 0$ & $1 / 0$ & IA 10 & 2 \\
\hline 8 & 7 & 2 & $1 / 0$ & $1 / 0$ & IA 4 & 3 \\
\hline
\end{tabular}

Note:-NIHSS indicates National Institutes of Health Stroke Scale; mRS, modified Rankin scale; IA, intra-arterial; rtPA, tissue plasminogen activation; TIMI, Thrombolysis in Myocardial Infarction Scores; MCA, middle cerebral artery; IV, intravenous.

of $22 \mathrm{mg}$. Patients were treated with only intra-arterial rtPA if they presented after 3 hours and within 6 hours of stroke onset. In patients proceeding to angiography, a $6 \mathrm{~F}$ catheter was inserted into the right common femoral artery with use of the Seldinger technique. Heparin was administered at the onset and during the procedure at the discretion of the interventionalist. A 5F diagnostic catheter was initially used for cerebral angiography. Occlusion of the symptomatic ICA was first confirmed before proceeding with angiography of other vessels to ascertain the status of Circle of Willis collaterals (PcomA and AcomA).

The most direct collateral route to the symptomatic occluded MCA was used for intra-arterial thrombolysis. The diagnostic catheter was exchanged for a $6 \mathrm{~F}$ guiding catheter, and a microcatheter was then advanced over a 0.014 -inch microguidewire through collateral vessels to the occluded MCA. Intra-arterial rtPA was then administered within the clot at a rate of 2 to $5 \mathrm{mg}$ for 5 minutes until recanalization was achieved or 20 to $25 \mathrm{mg}$ of rtPA was administered.

The degree of angiographic occlusion was assessed before and after treatment by use of Thrombolysis in Myocardial Infarction (TIMI) scores (graded as 0 for absent perfusion, 1 for minimal distal perfusion, 2 for partial perfusion, and 3 for complete perfusion). ${ }^{10}$

Noncontrast CT and CTA examinations were performed at 24 hours routinely in 3 patients. MR imaging and MRA were performed in 5 patients at 24 to 36 hours. Intracerebral hemorrhages were classified according to the European Cooperative Acute Stroke Study criteria as $\mathrm{PH} 1, \mathrm{PH} 2, \mathrm{HI} 1$, or $\mathrm{HI} 2{ }^{15}$

Clinical severity at baseline and at 24 hours after symptom onset was assessed with use of the National Institutes of Health Stroke Scale (NIHSS) conducted by neurologists who were fellows in stroke and were certified in the NIHSS scoring. The modified Rankin Scale (mRS) was used to assess clinical outcome at 30 and 90 days. Good outcome was defined as a mRS score of greater than or equal to 2 . Intracranial hemorrhage was considered as symptomatic if the patient had neurologic worsening of 4 points or more on the NIHSS and if the hemorrhage was likely to be the cause of neurologic deterioration.

\section{Results}

The clinical characteristics and imaging features of 8 patients with tandem ICA and MCA occlusions treated with intra-arterial thrombolysis via catheter navigation through intact collateral pathways are summarized in Table 1 . There were 7 men and 1 woman with a mean age of $57 \pm 4$ years (range, 42-71 years). The median baseline NIHSS score was 14 (interquartile range, 9-17), and mean time to treatment (initiation of intraarterial thrombolysis) was $292 \pm 44$ minutes from symptom onset. The average time from the start of intra-arterial treatment to the end of treatment was $48 \pm 18$ minutes. Early ischemic changes were detected in all patients, and median ASPECT score was 8 (range, 6-9). All patients had tandem ICA and proximal MCA occlusions on conventional angiogram.

Intravenous rtPA was given in 4 of the 8 patients within 3 hours of symptom onset, followed by administration of intraarterial rtPA via microcatheter navigation through the intact collateral pathways. Four of the 8 patients received only intraarterial rtPA (Table 2). Successful recanalization of the MCA (TIMI 2 or 3 ) was achieved in 6 of 8 patients (Table 2). Two of 8 patients did not demonstrate immediate MCA recanalization at the end of the procedure; however, one of these patients had complete recanalization of MCA at 24-hour follow-up CTA after stroke.

There were no complications during intra-arterial thrombolysis by use of the collateral intact pathways. Immediate postprocedural noncontrast CT scan of the head revealed no hemorrhage in any patient. Small petechial asymptomatic hemorrhages ( $\mathrm{PH} 1$ ) were identified in 3 patients on 24-hour 

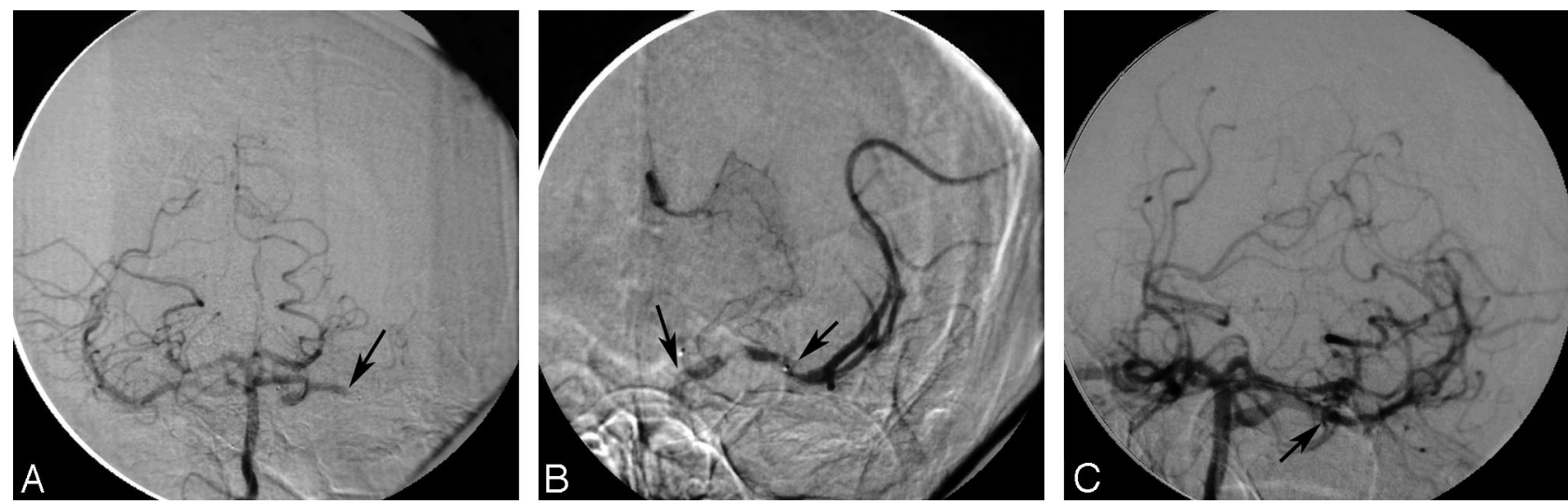

Fig 1. A, The right vertebral artery injection shows bilateral filling of the PcomA arteries that supply both internal carotid arteries. The left MCA M1 segment is occluded (small black arrow) $B$, Microcathether injection demonstrates a thrombus in the M1 segment (arrow) of the MCA. $C$, The right vertebral artery injection shows successful recanalization of the left MCA (arrow)

follow-up MR imaging or CT scans. We did treat 6 patients with antiplatelet therapy and 2 patients with anticoagulant therapy. Regarding the postprocedural management of patients with antithrombotics, we took our decision on case-bycase basis. In the presence of poor collaterals, partially recanalized MCA and ICA, and less extensive infracted tissue, we favored anticoagulant therapy. Follow-up CTA or MRA demonstrated recanalization of the ICA in 5 patients. A severe focal stenosis of the cervical ICA was detected in 3 of 5 patients. Neurologic worsening was not observed in any patient during hospitalization. Because of the high risk for recurrent stroke associated with a symptomatic, severely stenosed internal carotid artery, carotid endarterectomy was performed in these 3 patients within 2 weeks.

All patients had good outcomes with a median mRS of 1 (range, $0-2$ ) at 1 and 3 months after thrombolysis. There were no deaths or recurrent strokes during the 1 month of followup. Details of the outcomes are summarized in Table 2.

\section{Case Illustration 1}

A 64-year-old right-handed man presented to the emergency department with acute onset of aphasia, right-sided hemiplegia, and marked left-sided gaze preference, with a NIHSS score of 21. A CT scan of the head was performed within 1 hour of onset of the event and revealed a hyperattenuated MCA sign with a baseline ASPECT score of 8 . He was treated with $8.7 \mathrm{mg}$ of intravenous rtPA and taken to the angiography suite for possible intra-arterial therapy.

An arteriogram of the left common carotid artery revealed complete occlusion of the left ICA in the neck (Case 3 in Tables). There was no filling of the MCA territory. The right common carotid artery arteriogram demonstrated a complete occlusion of the right ICA in the neck. An arteriogram of the right vertebral artery showed bilateral filling of the posterior communicating arteries supplying both distal ICAs. The left M1 segment of the MCA was occluded (Fig 1A). A 6F guiding catheter was placed in the right vertebral artery. A Micro-Softstream (Boston Scientific, Natick, Mass) microcatheter was advanced (over a 0.014-inch microguidewire) into the basilar artery, then forward through the PcomA into the left supraclinoid ICA. The catheter was then advanced into the distal M1 segment. Superselective injection through the microcatheter demonstrated thrombus in the proximal MCA (Fig 1B). A total of $18 \mathrm{mg}$ of rtPA was administered into the thrombus for 10 minutes. Repeated arteriograms revealed complete resolution of the thrombus and significant restoration of flow (TIMI 3 ) in the left MCA (Fig 1C). The patient started to improve immediately and had marked improvement of his speech and strength by the following morning. MR imaging of the brain was performed 24 hours after thrombolysis and showed right frontal opercular, insular, and basal ganglia infarction. Carotid Doppler was done 5 days after thrombolysis and demonstrated recanalization of the previously occluded carotid artery in the neck with a trickle of flow through a severe stenosis. The patient underwent an uncomplicated left carotid endarterectomy. He was discharged with a near-complete recovery (mRS 1).

\section{Case Illustration 2}

A 65-year-old right-handed man presented to the emergency department with acute onset of severe right-sided hemiparesis, expressive aphasia, and pronounced right facial drop with a NIHSS score of 18 (Case 6). A noncontrast CT scan of the head showed subtle hypoattenuation in the left basal ganglia and insula. CTA revealed complete occlusion of the left cervical ICA with thrombus in the proximal left M1 segment of the MCA. En route to the angiography suite, $0.6 \mathrm{mg} / \mathrm{kg}$ (two thirds of the normal intravenous dose) of intravenous rtPA was administered. The arteriogram of the left common carotid artery demonstrated an irregular stump at the origin of the left ICA. There was no filling of the MCA territory. The left vertebral artery injection showed filling of the supraclinoid segment of the left ICA via the left PcomA. There was abrupt cutoff of the left MCA, consistent with embolic occlusion approximately 4 $\mathrm{mm}$ beyond the ICA bifurcation (Fig 2A). Through a 6F guiding catheter placed in the right distal common carotid artery, a microcatheter was positioned into the AcomA and $5 \mathrm{mg}$ of rtPA was administered. This did not result in any filling of the left M1 segment. The guiding catheter was repositioned within the left vertebral artery and the microcatheter advanced into the left M1 clot through the left PcomA. Contrast injection through the microcatheter showed M1 thrombus. A total dose of $10 \mathrm{mg}$ of rtPA was administered into the thrombus for 20 minutes. A control angiogram of the left vertebral artery revealed partial recanalization of the left M1 segment with reestablishment of flow within the inferior division of the left 

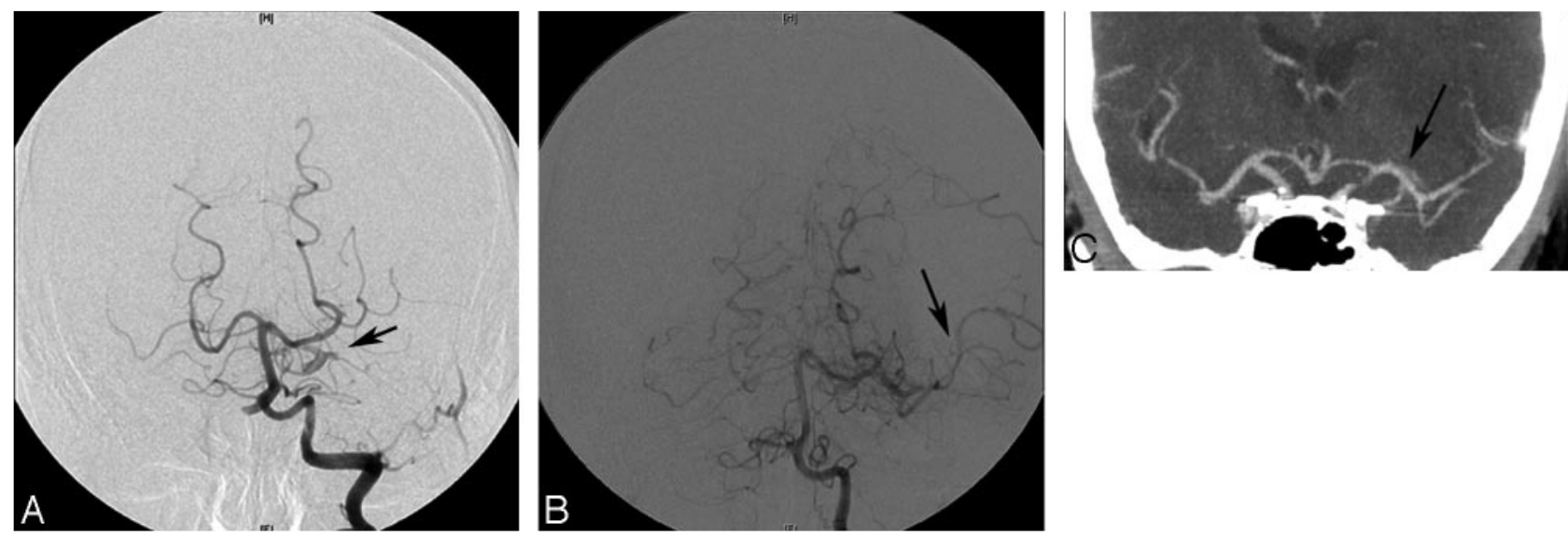

Fig 2. $A$, The left vertebral angiogram demonstrates an occlusion of the proximal MCA M1 segment (arrow). $B$, Administration of 15 mg of intra-arterial rtPA through a microcathether by use of the AcomA and PcomA arteries resulted in partial lysis of thrombus in the left M1 segment with restoration of flow within the inferior division of the left MCA. $C$, Follow-up CT angiogram 24 hours after stroke shows a complete recanalization of the M1 segment of the left MCA (arrow).

MCA (Fig 2B). A noncontrast CT of the head and CTA were performed 24 hours after thrombolysis, demonstrating left frontal opercular, insular, basal ganglia, and anterior internal capsule infarction and complete recanalization of the M1 segment of the left MCA (Fig 2C). On CTA, the left cervical ICA remained occluded. The patient was discharged with a favorable neurologic outcome (mRS 2) and did not have any recurrent stroke or transient ischemic events within 3 months.

\section{Discussion}

Extracranial ICA occlusion associated with MCA occlusion results in significant disability and death in $20 \%$ of patients. ${ }^{16}$ Furthermore, the presence of tandem cervical ICA and proximal MCA occlusion independently predicted poor recanalization and outcome. ${ }^{17}$ Tandem cervical ICA and proximal MCA occlusion can cause a decrease in regional cerebral perfusion pressure, which may hamper dissolution of an MCA clot after intravenous thrombolysis. ${ }^{18}$ Different endovascular approaches for acute stroke related to tandem cervical ICA and embolic MCA occlusions have been proposed to achieve better outcomes. However, there currently is no generally accepted treatment protocol.

The primary cause of symptoms and clinical outcome are mainly because of thrombus in the MCA rather than occlusion of the cervical ICA. ${ }^{16}$ The lenticulostriate arteries (LSAs) are functional end-arteries without collateral. Thrombus in the proximal segment of the MCA may lead to occlusion of the orifices of these arteries and cause cerebral infarction. ${ }^{19}$ Hence, the status of the LSAs and the length of time required for revascularization determines outcome. In the absence of sufficient leptomeningeal collaterals, expansion of the infarct core into surrounding hypoperfused tissue occurs earlier. Several published studies of intra-arterial thrombolytic therapy indicate that treatment initiated within 3 to 4 hours of symptom onset is associated with higher rates of recanalization and better outcome. ${ }^{20,21}$ Moreover, successful recanalization of ICA and MCA vessels may not reflect clinical outcome because of evolving cerebral infarction. Prompt reperfusion of initially still viable tissue and prevention of subsequent expansion of cerebral infarction are the critical factors to achieve a favorable neurologic outcome.

Traversing an acutely occluded cervical ICA to perform thrombolysis of MCA clot has been reported as effective therapy but may be time consuming and risky. ${ }^{22}$ Although microguidewires and catheters may easily transverse fresh thrombus in a recently occluded, blind probing with a microguidewire and manipulation of a microcatheter in an occluded vessel may lead to vessel dissection or perforation. The possibility also exists of entering the false lumen of an ICA dissection. Furthermore, dislodgement of thrombus while crossing the occluded cervical ICA is possible. Emergent stent placement or angioplasty of the occluded cervical ICA seems to have a high recanalization rate but also takes time to perform, during which brain infarction may be expanding. ${ }^{8,23} \mathrm{An}$ additional concern is the risk for hyperperfusion syndrome resulting in intracerebral hemorrhage. ${ }^{24}$

We report our experience in thrombolysis of occluded MCA by using collateral pathways in patients with tandem cervical ICA and MCA occlusion. Our first goal was to address the acute clot of the MCA rather than to traverse the occluded cervical ICA. Intra-arterial thrombolysis was given via the PcomA artery in 3 patients and the AcomA artery in 4 patients. In 1 patient, we used both PcomA and AcomA arteries to gain access to the MCA. The concern with advancing a microguidewire through Circle of Willis collaterals is the risk for dissection and embolic stroke. This risk may be higher in patients with advanced atherosclerotic stenoses in the proximal vertebral and carotid arteries. Fortunately, we did not experience this complication, and access of the posterior and anterior collateral routes was relatively straightforward. However, it is important to emphasize that it could be difficult to use this technique in the absence of intact collateral pathways including the PcomA and AcomA arteries. Hence, evaluation of collateral pathways by imaging modalities such as CTA or MRA may guide us to choose the most appropriate technique of endovascular therapy for patients with ischemic stroke with tandem ICA and MCA occlusion. All of our patients had good neurologic outcome at 1 and 3 months after acute stroke.

After the successful recanalization of MCA, one is faced with the management dilemma of the treatment of the occlusion of the proximal ICA. In the presence of poor leptomeningeal collaterals to the affected hemisphere, unsuccessful recanalization of the MCA and high NIHSS score despite thrombolytic therapy via the collateral pathways, immediate 
therapy of occluded ICA may be considered by angioplasty followed by stent placement. In our patients, good collateral flow was demonstrated despite persistent ICA occlusion, and consequently, attempts were not made to immediately recanalize the ICA. We did not observe any clinical deterioration during the hospitalization of our patients. Within days, spontaneous recanalization of the cervical ICA was seen in 5 of our patients. High-grade stenoses were present in 3 patients, who subsequently underwent carotid endarterectomy within 2 weeks of the stroke. ${ }^{25}$

\section{Conclusions}

There are no standardized treatment guidelines for intra-arterial treatment of acute tandem ICA or MCA occlusion. Time plays a critical factor in patient outcome. Use of collateral vessels to direct thrombolysis into the occluded MCA may provide an alternative quick, safe, and effective endovascular treatment.

\section{References}

1. El-Mitwalli A, Saad M, Christou I, et al. Clinical and sonographic patterns of tandem internal carotid artery/middle cerebral artery occlusion in tissue plasminogen activator-treated patients. Stroke 2002;33:99-102

2. Meyer FB, Sundt TM, Piepgras DG, et al. Emergency carotid endarterectomy for patients with acute carotid occlusion and profound neurological deficits. Ann Surg 1986;203:82-89

3. Furlan A, Higashida R, Wechsler L, et al. Intra-arterial prourokinase for acute stroke ischemic stroke. The PROACT II study: a randomized controlled trial. Prolyse in acute cerebral thromboembolism JAMA 1999;282:2003-11

4. Nesbit GM, Clark WM, O'Neill OR, et al. Intracranial intraarterial thrombolysis facilitated by microcathether navigation through an occluded cervical internal carotid artery. J Neurosurg 1996;84:387-92

5. Flint AC, Duckwiler GR, Budzik RF, et al. Mechanical thrombectomy of intracranial internal carotid occlusion: pooled results of the MERCI and Multi MERCI Part I trials. Stroke 2007;38:1274-80

6. Dabitz R, Triebe S, Leppmeier U, et al. Percutaneous recanalization of acute internal carotid artery occlusions in patients with severe stroke. Cardiovasc Intervent Radiol 2007;30:34-41

7. Takahisa T, Ken K, Mima T, et al. Balloon angioplasty for embolic total occlusion of the middle cerebral artery and ipsilateral carotid stenting in an acute stroke stage. AJNR Am J Neuroradiology 1999;20:1462-64

8. Jovin TG, Gupta R, Uchino K, et al. Emergent stenting of extracranial internal carotid artery occlusion in acute stroke has a high revascularization rate. Stroke 2005;36:2426-30
9. Lum C, Stys PK, Hogan MJ, et al. Acute anterior circulation stroke: recanalization using clot angioplasty. Can J Neurol Sci 2006;33:217-22

10. Chesebro JH, Knatterud G, Roberts R, et al. Thrombolysis in Myocardial Infarction (TIMI) Trial, Phase 1: A comparison between intravenous tissue plasminogen activator and intravenous streptokinase clinical findings through hospital discharge. Circulation 1987;76:142-54

11. Kole MK, Pelz DM, Lee DH, et al. Intra-arterial thrombolysis of embolic middle cerebral artery using collateral pathways. CJNS 2005;32:257-60

12. Restrepo L, Pradilla G, Llinas R, et al. Perfusion- and diffusion-weighted MR imaging-guided therapy of vertebral artery dissection: intra-arterial thrombolysis through an occipital vertebral anastomosis. AJNR Am J Neuroradiol 2003;24:1823-26

13. Pexman JH, Barber PA, Hill MD, et al. Use of the Alberta Stroke Program Early CT Score (ASPECTS) for assessing CT scans in patients with acute stroke. AJNR Am J Neuroradiol 2001;22:1534-42

14. Adams H, Adams R, Del Zoppo G, et al. Guidelines for the early management of patients with ischemic stroke: 2005 guidelines update. A scientific statement from the Stroke Council of the American Heart Association/American Stroke Association. Stroke 2005;36:916-23

15. Berger C, Fiorelli M, Steiner T, et al. Hemorrhagic transformation of ischemic brain tissue: asymptomatic or symptomatic? Stroke 2001;32:1330-35

16. Adams HP, Bendixen BH, Leira E, et al. Antithrombotic treatment of ischemic stroke among patients with occlusion or severe stenosis of the internal carotid artery: a report of the Trial of Org 10172 in Acute Stroke Treatment (TOAST). Neurology 1999;53:122-25

17. Rubiera M, Ribo M, Delgado-Mederos R, et al. Tandem internal carotid artery/ middle cerebral artery ccclusion. An independent predictor of poor outcome after systemic thrombolysis. Stroke 2006;37:2301-05

18. Rubiera M, Alvarez-Sabin J, Ribo M, et al. Predictors of early arterial reocclusion after tissue plasminogen activator-induced recanalization in acute ischemic stroke. Stroke 2005;36:1452-56

19. Donnan G, Norrving B, Bamford J, et al. Striocapsular Infarcts. Oxford, UK Oxford University Press; 2002:195-208

20. Suarez JI, Sunshine JL, Tarr R, et al. Predictors of clinical improvement, angiographic recanalization, and intracranial hemorrhage after intra-arterial thrombolysis for acute ischemic stroke. Stroke 1999;30:2094-100

21. Zaidat OO, Suarez JI, Santillan C, et al. Response to intra-arterial and combined intravenous and intra-arterial thrombolytic therapy in patients with distal internal carotid artery occlusion. Stroke 2002;33:1821-27

22. Srinivasan A, Goyal M, Stys $P$, et al. Microcathether navigation and thrombolysis in acute symptomatic cervical internal carotid occlusion. AJNR Am J Neuroradiology 2006;27:774-79

23. Spearman MP, Jungreis CA, Wechsler LR. Angioplasty of the occluded internal artery. AJNR Am J Neuroradiology 1995;16:1791-96

24. Meyers PM, Higashida RT, Phatouros CC, et al. Cerebral hyperperfusion syndrome after percutaneous transluminal stenting of the craniocervical arteries. Neurosurgery 2000;47:335-45

25. Endo S, Hirashima Y, Kurimoto M, et al. Acute pathological features with angiographic correlates of the nearly or completely occluded lesions of the cervical internal carotid artery. Surg Neurol 1996;46:222-28 